\title{
Post-operative rehabilitation after PIP joint arthroplasty with early active motion: a retrospective review of outcomes
}

\author{
A. L. Pratt MSc, DipCOT \\ School of Health Sciences and Social Care, Brunel University, Uxbridge, UK \\ N. Burr MSc, Grad Dip Phys. \\ Hand Therapy Unit, Mount Vernon Hospital, Northwood, UK
}

[abstract]

We present a retrospectively review of outcomes of the first fifteen patients who underwent proximal interphalangeal (PIP) joint arthroplasty and were treated using the same early active motion rehabilitation regime introduced by the therapy department at Mount Vernon Hospital. The regime utilises early motion of the PIP joint while protecting the arthroplasty with a small static splint and digit strapping to reduce lateral forces on the joint.

The notes of fifteen patients were reviewed and their outcomes presented. To evaluate the outcomes in more detail the patients were divided into three groups according to their diagnostic reason for the procedure (rheumatoid arthritis, osteoarthritis and trauma). The overall mean arc of motion at the PIP joint on discharge from therapy was $54^{\circ}$ improved from $28^{\circ}$ pre operatively.

The patients with the osteoarthritic PIP joints gained the largest improvement in the PIP joint arc of motion and required the least rehabilitation intervention. Patients with rheumatoid arthritis required intense rehabilitation to gain less overall PIP joint motion 
but still reported satisfaction with their outcome. All 15 patients experienced an improvement in their pain level and subjectively reported increased function in their affected hand.

Following this retrospective review of cases the team continue to use this regime for metal and silastic prosthesis but now routinely provide additional written information pre operatively to assist patients' understanding of the procedure and the extent of the rehabilitation required. 


\section{INTRODUCTION}

Movement and function of the hand are dependent upon pain free and mobile joints with $34-39 \%$ of the total arc of digital motion occurring at the proximal interphalangeal joint (PIP joint) (Jones and Stern 1994). The PIP joint is frequently involved in rheumatoid arthritis (RA), degenerative osteoarthritis (OA) and trauma causing pain, weakness, malalignment and reduced motion. This often reduces function to the point of requiring surgical treatment (Linschield et al1997, Swanson et al 1985).

Green et al (1991) and Pellegrini and Burton (1990) have suggested that to achieve a more stable pain free PIP joint (mainly for the index and middle finger) allowing better pinch and precision grip an arthrodesis of the joint in flexion should be the surgical treatment of choice. The ring and little fingers require mobility for good hand function so joint arthroplasty is often considered for these digits (Swanson and De Groot Swanson, 1994). PIP joint arthroplasty is still used widely in practice for all fingers as maintaining mobility of the PIP joint can also enhance the mobility of the adjacent fingers (Green et al 1991, Linscheid et al1997).

Since the first synthetic prosthesis was introduced in 1940 (Burman, 1940) many different types of prosthesis have been available for PIP joint arthroplasties. To date most of the literature on PIP joint arthroplasty continues to present positive outcomes of pain relief and increased joint mobility (Ashworth et al 1997, Conolly and Rath 1991, Green et al 1991, Hage et al 1999, Lin et al 1995, Strickland et al1982, Swanson et al 1985). Green et al (1991) state that a PIP joint arthroplasty provides and active arc of motion of approximately $60^{\circ}$ at the PIP joint. 
This range of motion (ROM) is often not achieved in practice as the pre-operative PIP joint deformity greatly influences the overall outcome (Ashworth et al 1997, Conolly and Rath 1991, Lin et al 1995, Linscheid et al 1997, Strickland et al 1982). Despite an abundance of literature few articles described the post-operative rehabilitation regimen and the difficulties that hand therapists have to overcome when rehabilitating this anatomically complex joint.

The aim of this paper is therefore to present the rehabilitation regime which has been used over the last six years at Mount Vernon Hospital (West Hertfordshire NHS Trust), to discuss the difficulties found during the rehabilitation of the first 15 patients treated according to this regime and to present their outcomes. The regime was utilised irrespective of the surgeon's choice of prosthesis (i.e. metal/silastic). A lateral surgical approach was utilised within this unit to protect the extensor mechanism during surgery. None of the 15 patients underwent any reconstruction of their central slip or soft tissue, which would have prevented the use of this early motion regime.

\section{REHABILITATION GUIDELINE USED}

\section{$\underline{\text { Pre-operative assessment }}$}

Pre operative assessment undertaken as part of the routine pre-operative management included:

- ROM using a goniometer (Adams et al. 1999),

- Deformity of their digit (swan neck deformity, boutonnière deformity or deviation of the PIP joint)

- Pain using a visual analogue scale (Revill et al 1976) 
- Patient self-reporting of functional difficulties (subjectively).

This enables the hand therapist to establish a baseline assessment of patient's difficulties and informs the therapist of factors that may influence postoperative treatment.

\section{$\underline{\text { Post-operative rehabilitation regimen }}$}

Following surgery all patients are seen within 72 hours to commence the early motion rehabilitation regime. Dressings are reduced and an extension splint fabricated to maintain the PIP joint in alignment and maintain/increase extension as necessary (Figure 1). The splint immobilises the affected and neighbouring digit to provide maximum support to the PIP joint at rest and is reviewed regularly. We have found that in clinical practice maximum PIP joint extension may take a few weeks to achieve in patients who have a pre-operative boutonnière deformity, flexion contracture or excessive postoperative oedema

\section{Figure 1 A Bedford splint worn with an extension splint}


This splint is worn continually for the first four weeks to allow the soft tissues to heal and inflammation to settle. The patient is advised to remove the splint hourly to commence controlled early motion of the affected digits. A Bedford splint or buddy strapping is also utilised during exercises to protect the PIP joint arthroplasty, from external lateral forces, which make it unstable. Patients are advised regarding the lack of stability of this joint especially within the early rehabilitation period. The Bedford splint is the preferred choice of protection as it also assists in reducing PIP joint oedema. Occasionally this is not possible in the first couple of weeks post operatively due to wound concerns and buddy strapping is used.

The following exercises are advised to be performed ten times each hour: -

- Active flexion and extension of metacarpophalangeal (MCP) joints

- Active flexion of the PIP joint (important for patients who have had a swanneck-deformity pre-operatively)

- Active extension of the PIP joint (important for patients who have had a boutonnière deformity or flexion contracture pre-operatively)

- Active flexion and extension of PIP and distal interphalangeal (DIP) joints

- Mass flexion and extension of digits.

- Active ROM of all unaffected joints

Patients are also advised to elevate the hand and avoid use of the affected fingers/s in light functional activities for at least four weeks.

At two to three weeks post-operatively passive flexion and extension exercises are introduced if motion is difficult to achieve actively and inflammation and pain are 
subsiding. Scar massage is commenced following the removal of stitches and Coban wrap is regularly used for those patients who experience excessive oedema.

At four weeks post-operatively patients are advised on light functional activities (e.g. fastening buttons, handling money) within their protective Bedford splint/buddy strapping. Rotational movements or twisting activities are avoided.

From six weeks post-operatively function and grip strength are gradually increased through the use of graded activities provided that subjective pain and joint stability on examination have improved. The Bedford splint, or buddy strapping is continued if the PIP joint does not feel stable upon assessment. Flexion strapping is utilised in patients who have not gained additional passive flexion. Functional activities involving rotational forces (i.e. opening jars, turning taps) are discouraged with the affected hand for at least 12 weeks post-operatively. This is longer than that recommended period by Swanson and Swanson De Groot (1994) who advocate strapping for only six weeks but in practice a longer period has been beneficial. The patient is weaned off the extension splint after eight weeks post-surgery depending on the residual extension deficit.

\section{REVIEW OF CASE SERIES}

The notes from the first fifteen patients ( 21 joints)treated by this regimen were reviewed to evaluate the outcomes achieved and with the aim of informing future therapy practice. All patients were reported to have found the rehabilitation regime easy to follow and had regularly attended for follow-up with no post-operative complications. 
Seven patients (12 joints) who had PIP joint arthroplasty had a diagnosis of RA, of which four patients had swan neck deformities. One patient had no active PIP joint ROM pre operatively. Three patients had deviated PIP joints affecting their motion and function.

Five patients had degenerative OA. Of these two patients had fixed flexion deformities, two patients had boutonnière deformities and one patient had ulnar deviation of the PIP joint.

Three patients required surgical intervention following previous trauma of the PIP joint with extensor mechanism damage. Of these, two complained of reduced motion and a boutonnière deformity and one had reduced motion and a deviated PIP joint.

The distribution of PIP joints involved was two index, nine middle, five ring and five little fingers. The dominant hand was involved in nine patients. The mean patient age was 57 (range 45-73) years, five patients were male and ten were female.

\section{$\underline{\text { Range of motion }}$}

ROM was reviewed from the baseline pre-operative assessment and again at the time of discharge from hand therapy.

Table 1 shows the mean flexion, extension and arc of motion of the PIP joint gained by the 15 patients. Overall the arc of motion improved from a mean of $28^{\circ}$ pre operatively to $54^{\circ}$ at discharge from therapy. 
Table 1 Mean extension, flexion and arc of motion of the PIP joint

\begin{tabular}{|l|l|l|}
\hline Mean motion & $\begin{array}{l}\text { Pre-op }\left(^{\circ}\right) \\
(\mathrm{n}=21)\end{array}$ & $\begin{array}{l}\text { Discharge } \\
\text { from therapy } \\
\left({ }^{\mathbf{o}}\right)(\mathrm{n}=21)\end{array}$ \\
\hline Extension & $\begin{array}{c}\mathbf{6} \\
(\mathrm{r}=-30 \text { to } 95)\end{array}$ & $\begin{array}{c}\mathbf{4} \\
(\mathrm{r}=-42 \text { to } 72)\end{array}$ \\
\hline Flexion & $\begin{array}{c}\mathbf{3 4} \\
(\mathrm{r}=-30 \text { to } 85)\end{array}$ & $\begin{array}{c}\mathbf{5 8} \\
(\mathrm{r}=-16 \text { to } 94)\end{array}$ \\
\hline Arc & $\begin{array}{c}\mathbf{2 8} \\
(\mathrm{r}=0 \text { to } 71)\end{array}$ & $\begin{array}{c}\mathbf{5 4} \\
(\mathrm{r}=3 \text { to } 103)\end{array}$ \\
\hline
\end{tabular}

The data on motion were analysed according to the patients' diagnostic group (RA OA and trauma) (Tables 2, 3 and 4).

Table 2 Mean motion achieved by the RA patients

\begin{tabular}{|l|c|c|}
\hline Mean motion & $\begin{array}{c}\text { Pre-op }\left(^{\circ}\right) \\
(\mathrm{n}=12)\end{array}$ & $\begin{array}{c}\text { Discharge } \\
\text { from therapy } \\
\left({ }^{\circ}\right)(\mathrm{n}=12)\end{array}$ \\
\hline Extension & $\begin{array}{c}\mathbf{- 9} \\
(\mathrm{r}=18 \text { to }-30)\end{array}$ & $\begin{array}{c}\mathbf{- 5} \\
(\mathrm{r}=41 \text { to }-42)\end{array}$ \\
\hline Flexion & $\begin{array}{c}\mathbf{1 8} \\
(\mathrm{r}=-30 \text { to } 61)\end{array}$ & $\begin{array}{c}\mathbf{3 2} \\
(\mathrm{r}=-16 \text { to } 88)\end{array}$ \\
\hline Arc & $\begin{array}{c}\mathbf{2 4} \\
(\mathrm{r}=0 \text { to } 71)\end{array}$ & $\begin{array}{c}\mathbf{3 8} \\
(\mathrm{r}=3 \text { to } 103)\end{array}$ \\
\hline
\end{tabular}

Table 3 Mean motion achieved by the OA patients

\begin{tabular}{|l|c|c|}
\hline Mean motion & $\begin{array}{c}\text { Pre-op }\left(^{\circ}\right) \\
(\mathrm{n}=8)\end{array}$ & $\begin{array}{c}\text { Discharge } \\
\text { from therapy } \\
\left({ }^{\circ}\right)(\mathrm{n}=8)\end{array}$ \\
\hline Extension & $\begin{array}{c}\mathbf{3 0} \\
\text { (r=95 to } 10)\end{array}$ & $\begin{array}{c}\mathbf{1 8} \\
(\mathrm{r}=72 \text { to }-3.0)\end{array}$ \\
\hline Flexion & $\begin{array}{c}\mathbf{5 4} \\
(\mathrm{r}=30 \text { to } 100)\end{array}$ & $\begin{array}{c}\mathbf{8 3} \\
(\mathrm{r}=73 \text { to } 94)\end{array}$ \\
\hline Arc & $\begin{array}{c}\mathbf{2 4} \\
(\mathrm{r}=3.0 \text { to } 49)\end{array}$ & $\begin{array}{c}\mathbf{6 5} \\
(\mathrm{r}=14 \text { to } 91)\end{array}$ \\
\hline
\end{tabular}


Table 4 Mean motion achieved by the trauma patients

\begin{tabular}{|l|c|c|}
\hline Mean motion & $\begin{array}{c}\text { Pre-op }\left(^{\circ}\right) \\
(\mathrm{n}=3)\end{array}$ & $\begin{array}{c}\text { Discharge } \\
\text { from therapy } \\
\left({ }^{\circ}\right)(\mathrm{n}=3)\end{array}$ \\
\hline Extension & $\begin{array}{c}\mathbf{2 1} \\
(\mathrm{r}=42 \text { to }-5)\end{array}$ & $\begin{array}{c}\mathbf{2 6} \\
(\mathrm{r}=54 \text { to }-2)\end{array}$ \\
\hline Flexion & $\begin{array}{c}\mathbf{5 9} \\
(\mathrm{r}=45 \text { to } 85)\end{array}$ & $\begin{array}{c}\mathbf{7 8} \\
(\mathrm{r}=67 \text { to } 90)\end{array}$ \\
\hline Arc & $\begin{array}{c}\mathbf{3 8} \\
(\mathrm{r}=19 \text { to } 51)\end{array}$ & $\begin{array}{c}\mathbf{5 2} \\
(\mathrm{r}=22 \text { to } 92)\end{array}$ \\
\hline
\end{tabular}

The patients with RA experienced a lower mean increase in their arc of motion $\left(24^{\circ}\right.$ pre operatively to $38^{\circ}$ at discharge). A closer look at individual results showed that 11 out of the 12 RA joints increased their arc of motion post operatively although this was not by a large proportion. The two patients with the significant pre-operative swan neck deformity found it very hard to gain and maintain their flexion post-operatively and required more intensive therapy than the others. Even though the arc of motion for this patient group was small they subjectively reported improvement in function and satisfaction post-operatively was high. Patients who no longer had a swan neck deformity commented on the improved cosmetic appearance of their hand and welcomed that it was no longer necessary to wear swan neck prevention splints.

The patients with OA experienced the largest increase in arc of motion from a mean of $24^{\circ}$ pre-operatively to $65^{\circ}$ at discharge. This patient group achieved their outcomes with less rehabilitation time than the other two diagnostic groups.

The three trauma patients all improved their arc of motion (mean $38^{\circ}$ to $52^{\circ}$ at discharge) but the two with extensive damage to their extensor mechanism at the time of trauma required more intensive therapy and longer splintage to maintain the 
improvements gained after surgery. The patient without the boutonnière deformity preoperatively gained by far the best arc of motion $\left(92^{\circ}\right)$ in a shorter time period.

\section{$\underline{\text { Pre-operative PIP joint deviation }}$}

At the pre operative assessment five patients were found to have abnormal radial or ulnar deviation of the PIP joint (mean $24^{\circ}$, range $7^{\circ}$ to $39^{\circ}$ ). At discharge this had improved to $0^{\circ}$ for all patients. Patients with deviation of the PIP joint pre-operatively were advised to wear the Bedford splint or buddy strapping for longer periods postoperatively until their PIP joint was assessed to be more stable. The largest improvement in PIP joint deviation was from $39^{\circ}$ to $0^{\circ}$ in a 71 -year-old male with RA. All patients were extremely satisfied with the correction of their deviation, which they subjectively reported helped improve function and cosmesis.

\section{$\underline{\text { Pain }}$}

At the pre-operative assessment all 15 subjects reported pain in their PIP joint with the exception of a 71-year-old male with a diagnosis of RA. The mean pain score on the visual analogue scale was four (range $=$ zero to nine) pre-operatively which improved to two (range=zero to three) at discharge from therapy.

\section{$\underline{\text { Intensity of rehabilitation required }}$}

The required post-operative therapy intervention varied with each individual case.

Patients were only discharged when ROM, realignment and pain were assessed to have improved or reached a plateau. However, even with this small patient group there was a trend observed that some diagnostic groups generally required more intensive therapy 
over a longer period. Figure 2 illustrates that for these 15 patients those with a diagnosis of RA required three times more rehabilitation sessions following their PIP joint arthroplasty than those with OA and over twice as much as those patients following trauma. This has led to more specific pre-operative advice being given to patients about the anticipated extent of the therapy required.

Figure 2 Mean therapy sessions for each diagnostic group

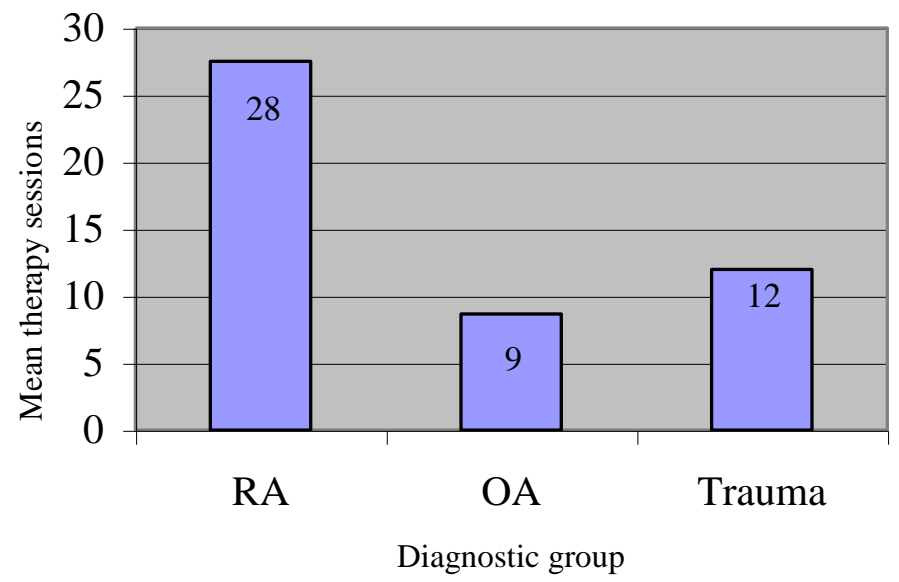

\section{DISCUSSION}

This review of fifteen cases revealed that the mean arc of motion of the PIP joint for the 15 patients using this simple rehabilitation regime was $54^{\circ}$ at discharge. The early active motion regime allows patients to gain good PIP joint motion whilst preventing complications occurring with other digits. Issues arising from pre-operative deformities can also be addressed early.

It is not possible to directly compare these outcomes with the results from other published reviews due to the lack of information regarding the rehabilitation regime, the splintage used and the differing follow up periods. 
Schneider (1991) reported an average arc of PIP joint motion of $48^{\circ}$ in 20 patients using a more complicated dynamic extension splint. Herren and Simmen (2000) reported a similar overall average mean ROM of $51^{\circ}$ in 38 patients but did not discuss any aspect of the post operative rehabilitation

Most papers reporting the results of PIP joint arthroplasty do not report on pre-operative deformities and tend to only state the diagnostic group of the patient (i.e. RA, OA, trauma). However, as reported by Swanson et al. (1985), and illustrated in these results, the pre-operative deformity can greatly influence the outcome that the patient can expect to achieve. This should be included in the pre-operative advice and education given to patients on rehabilitation periods and realistically achievable outcomes (Johnstone, 2001).

This review confirms that those patients with RA did not achieve a large change in their arc of motion. However the subjective report by these patients on pain relief, functional improvements and satisfaction from this surgical procedure and rehabilitation should not be undervalued.

The results illustrate that the largest improvements in arc of motion were gained by patients with $\mathrm{OA}\left(65^{\circ}\right)$, who achieved these results with less rehabilitation input than the other two groups. Pellegrini and Burton (1990) reported the findings of silicone prosthesis with the majority of OA patients achieving an average arc of motion of $56^{\circ}$. 
Patients who had a large pre-operative flexion contracture or boutonnière deformity generally required night extension splinting beyond six weeks post operatively and additional exercises for strengthening PIP joint extension. These patients also needed extra exercises and strapping to overcome the pre-operative DIP joint tightness.

For those patients who had a pre operative hyper-extended PIP joint (swan neck deformity) some form of passive flexion strapping was generally required to maximise flexion of the PIP and DIP joint alongside intensive exercises to increase flexor tendon pull through. Therefore the pre operative status or deformity of the joint affected the postoperative rehabilitation programme as discussed by Ashworth et al. (1997).

Connolly and Rath (1991) state that relief of pain and correction of deformity makes it a worthwhile operation even if the arc of motion is minimum. The literature (Heren and Simmen 2000, Lin et al 1995, Swanson et al. 1985,) reports that this procedure provides good pain relief for the majority of patients and this was also the case for these 15 patients.

This paper details the rehabilitation regime used and the patients' outcomes achieved on discharge from therapy. A longer period of follow up after discharge from therapy would be useful to determine if patients maintained these improvements longer term. The review of these 15 cases highlights some of the issues, which affected the outcomes and rehabilitation of these patients, however limitations must be acknowledged . Comparisons between different types of prosthesis or surgical procedure were not possible. Further research to evaluate the relative effectiveness of different types of 
rehabilitation regimens utilised after this surgical procedure is needed. The assessment of outcome also needs to include a more objective method to assess functional change. Following this case review the rehabilitation regime continues to be utilised at this Unit after PIP joint arthroplasty irrespective of the type of arthroplasty used. Changes to provide patients with more pre operative information have been made so they have a greater understanding of the rehabilitation requirements and their potential outcomes. 


\section{REFERENCES}

Adams L, Greene L, Topoozian E. Clinical Assessment Recommendations, Churchill Livingstone, New York, 1999: 55-70.

Ashworth C.R, Hansraj K.K, Todd A.O. et al. (1997) Swanson proximal interphalangeal joint arthroplsasty in patients with rheumatoid arthritis. Clinical Orthopaedics and Related Research 342: 34-37.

Burman M.S. (1940) Vitallium cap arthroplasty of metacarpophalangeal and interphalangeal joints of the fingers. Bull Hosp Joint Disease 1:79-89.

Conolly W.B and Rath S. (1991) Silastic implant arthroplasty for post traumatic stiffness of the finger joints. Journal of Hand Surgery. 16B (3): 286-292.

Green S.M, Posner M.A, Garay A. (1991) Silicone rubber arthroplasty of the proximal interphalangeal joint:Dorsal and lateral approaches. Seminars in Arthroplasty. 2(2) 130-138.

Hage J, Yoe E, Zevering J, Groot P (1999) Proximal Interpahalangeal Joint Silicone Arthroplasty for Posttrauamtic Arthritis. Journal of Hand Surgery 24A:73-77. 
Herren D.B. and Simmen B.R (2000) Palmar approach in flexible implant arthroplasty of the proximal interphalangeal joint. Clinical Orthopaedics and Related Research. 371:131-135.

Jones B.F and Stern P. J (1994). Interphalangeal joint arthrodesis. Hand Clinics 10 (2): 267-384.

Johnstone B.R. (2001) Proximal interphalangeal joint surface replacement arthroplasty. Hand Surgery 6(1): 1-11.

Lin H.H, Wyrick J.D, Stern P.J (1995) Proximal interphalangeal joint silicone replacement arthroplasty:clinical results using an anterior approach. Journal of Hand Surgery 20A(1): 123-132.

Linscheid R.L, Murray P.M, Vidal M, Beckenbaugh R.D. (1997) Development of a surface replacement arthroplasty for proximal interphalangeal joints. The Journal of Hand Surgery 22A (2): 886-298.

Pellegrini V.D, Burton R. I (1990) Osteoarthritis of the proximal interphalangeal joint of the hand : arthroplasty or fusion? Journal of Hand Surgery 15A: 194-209.

Revill S.I, Robinson J.O., Rosen M, Hogg M.I (1976): The reliability of a linear analogue for evaluating pain. Journal of Anaesthesia. 31: 1191-1198. 
Schnieder L. H. (1991) Proximal interphalangeal joint arthroplasty: the volar approach. Seminars in Arthroplasty 2 (2) :139-147.

Strickland J.W, Dustman J.A, Stelzer L et al. (1982) Post traumatic arthritis of the proximal interphalangeal joint.. Analysis of 100 silicone implants in articular fracture, fracture dislocation and crush injury. OrthopaedicReview 11 (8): 75-83.

Swanson A.B, Maupin B.K, Gajjar N.V, Swanson G. (1985) Flexible implant arthroplasty in the proximal interphalangeal joint of the hand. The Journal of Hand Surgery 10A (6): 796-805.

Swanson A.B. and De Groot Swanson G. (1994) Flexible implant resection arthroplasty of the proximal interphalangeal joint. Hand Clinics 10(2): 261-267. 